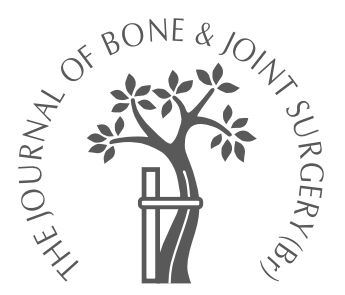

\title{
Anatomy of pilon fractures of the distal tibia
}

\section{J. Topliss, M. Jackson, R. M. Atkins}

From Bristol Royal Infirmary, Bristol, England
C. J. Topliss, FRCS, Specialist Registrar M. Jackson, FRCS (Tr \& Orth), Consultant Senior Lecturer

R. M. Atkins, DM, FRCS, Consultant Reader

University Department of Orthopaedic Surgery, Level 5, Bristol Royal Infirmary, Bristol BS2 8HW, UK.

Correspondence should be sent to Miss C. Topliss at 6 Broncksea Road, Bristol BS7 OSE, UK; e-mail:

toplisscj@doctors.org.uk

(C)2005 British Editorial Society of Bone and Joint Surgery doi:10.1302/0301-620X.87B5. $15982 \$ 2.00$

$J$ Bone Joint Surg $[B r]$ 2005;87-B:692-7.

Received 8 September 2004; Accepted 8 October 2004

In a series of 126 consecutive pilon fractures, we have described anatomically explicable fragments. Fracture lines describing these fragments have revealed ten types of pilon fracture which belong to two families, sagittal and coronal. The type of fracture is dictated by the energy of injury, the direction of the force of injury and the age of the patient.

A pilon fracture of the tibia involves the horizontal articular surface of the distal tibia with proximal extension. The injury is usually caused by a high-energy axial force which also produces severe soft-tissue damage. For an optimal outcome to be achieved there should be anatomical reconstruction of the joint, restoration of tibial alignment and stabilisation of the fracture to facilitate union. However, open surgery is associated with poor wound healing, restoration of the anatomy is difficult and delayed union and infection are common. This has led to the use of closed techniques which do not allow accurate reduction of the joint. Understanding of the anatomy of the fracture should allow the development of improved operative techniques and outcomes. To this end we have studied a large series in order to define the anatomy of the fracture.

\section{Patients and Methods}

Between 1993 and 2002, 122 patients (126 fractures) presented to our unit (Table I) either as acute admissions or tertiary referrals. Initial assessment included plain radiography. Open fractures were debrided and stabilised by a spanning external fixator within six hours. Before definitive fixation, CT was carried out to allow pre-operative planning. The plain radiographs and CT scans were analysed using a computer-aided design program (TurboCad, IMSI, Novato, California). The clinical details and injury data were collected from case notes and radiographs (Table I). Forty-one patients $(34 \%)$ had associated injuries.

Metaphyseal/diaphyseal dissociation was categorised as simple or comminuted and its level as metaphyseal, junctional or diaphyseal, according to the 'rule of squares'. ${ }^{1}$ Coronal and sagittal alignment was assessed. The fracture of

\begin{tabular}{|c|c|}
\hline Male: female & 93:33 \\
\hline $\begin{array}{l}\text { Age in yrs at injury } \\
\text { (range; SD) }\end{array}$ & $\begin{array}{l}45.3 \\
\text { (19 to } 77 ; 14)\end{array}$ \\
\hline \multicolumn{2}{|l|}{ Mechanism of injury } \\
\hline Fall in $\mathrm{ft}$ (geometric mean) & $84(9)$ \\
\hline Road-traffic accident & 24 \\
\hline Other & 18 \\
\hline \multicolumn{2}{|l|}{ Open fracture grade ${ }^{11,12}$} \\
\hline I & 1 \\
\hline II & 1 \\
\hline IIla & 9 \\
\hline IIIb & 24 \\
\hline \multicolumn{2}{|l|}{ Rüedi and Allgöwer ${ }^{7}$} \\
\hline I & 33 \\
\hline II & 38 \\
\hline III & 47 \\
\hline \multicolumn{2}{|l|}{ AO classification ${ }^{13}$} \\
\hline B2 & 2 \\
\hline B3 & 9 \\
\hline $\mathrm{C} 1$ & 5 \\
\hline $\mathrm{C} 2$ & 35 \\
\hline C3 & 67 \\
\hline
\end{tabular}

the fibula was classified according to DanisWeber. ${ }^{2}$ Any disruption of the syndesmosis and talofibular relationship was recorded.

The CT scan identified articular fragments and patterns. Fracture lines outlining the fragments were described with reference to the intermalleolar axis ${ }^{3,4}$ and the angle of the main fracture line was measured with respect to this. The term 'sagittal' described the plane perpendicular to, and 'coronal', the plane parallel to the intermalleolar axis. The CT scan also identified the relationship between the tibia and fibula and whether the anterior and posterior inferior tibiofibular ligaments had been ruptured.

All measurements were made three times and the mean recorded. After description of the types of fracture, the reproducibility was 


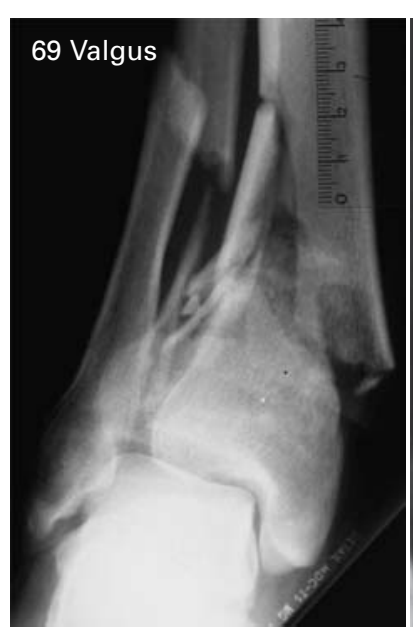

Fig. 1a

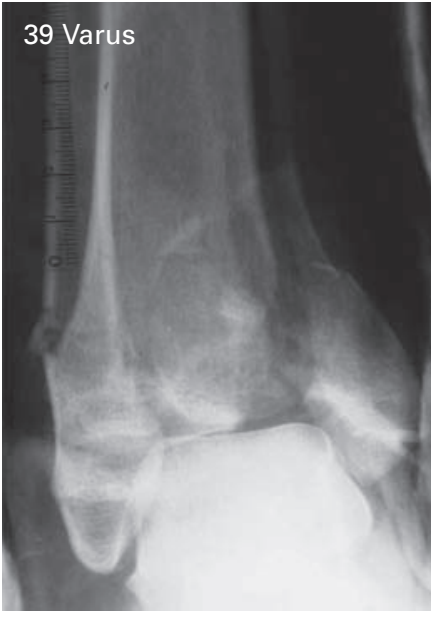

Fig. $1 b$

Radiographs showing pilon fractures in a) valgus and b) varus with the incidence in our series.

independently assessed by five orthopaedic surgeons. Kappa statistics ${ }^{5}$ were calculated for both inter- and intraobserver agreement. The values were averaged to estimate the level of agreement. ${ }^{6}$

Statistical analysis. This was carried out using StatView (SAS Inc, Cary, North Carolina), STATA (STATA Corporation College Station, Texas) and SPSS (SPSS Inc, Chicago, Illinois) software.

\section{Results}

Plain radiography. Before intervention 108 AP radiographs and 110 lateral radiographs were available. Most fractures lay in valgus $(69 ; 64 \%)$ and the remainder in varus $(39$; $36 \%)$ (Fig. 1). The lateral radiographs showed 65 (59\%) fractures with no angulation, $29(26 \%)$ with the apex posterior and $16(14 \%)$ with the apex anterior.

The metaphyseal/diaphyseal dissociation was simple in $47(40 \%)$ and comminuted in $71(60 \%)$ cases; $54(46 \%)$ were metaphyseal, $47(40 \%)$ crossed the metaphyseal/diaphyseal junction and $17(14 \%)$ were diaphyseal. The proximal and distal extents of the metaphyseal/diaphyseal dissociation were measured. The maximal proximal extent was $161 \mathrm{~mm}$ from the plafond (median $69 \mathrm{~mm}$, minimum $17 \mathrm{~mm}$ ) and the median distal extent was $24 \mathrm{~mm}$ (0 to 110) from the plafond. The centre of the metaphyseal/diaphyseal dissociation was at a median of $35.5 \mathrm{~mm}$ from the plafond ( 3 to 127$)$. In $24(20 \%)$ the fractures of the medial malleolus were below the plafond. The medial collateral ligament was ruptured in two of the 118 fractures.

Whole-length fibular views were available in 124 cases, of which 104 showed fractures. Most (75) were Weber type $\mathrm{C}$ of which five were segmental. The remainder were usually Weber type B (26) with only three being Weber type A.

There was only one ligamentous diastasis (Fig. 2a) but in 52 cases $(44 \%)$ there were separated lateral tibial fragments

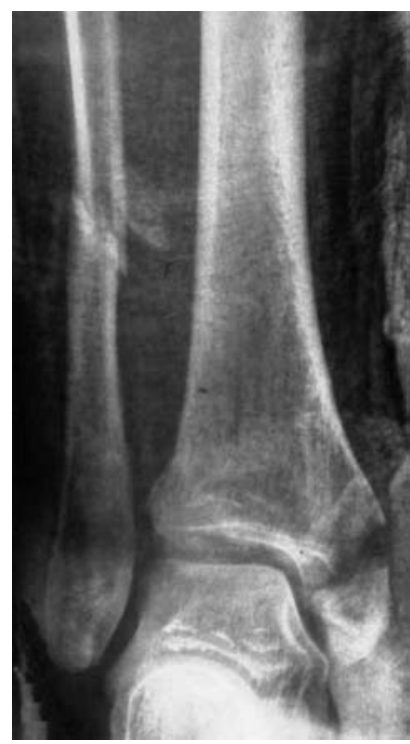

Fig. $2 a$

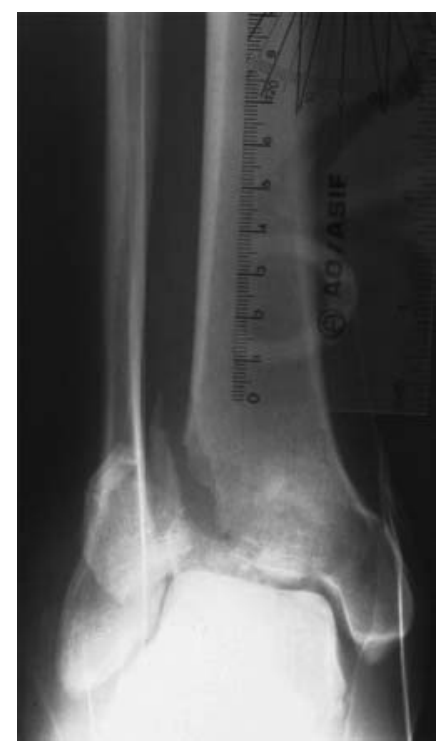

Fig. $2 b$
Radiographs showing a) ligamentous and b) functional diastases in pilon fractures.

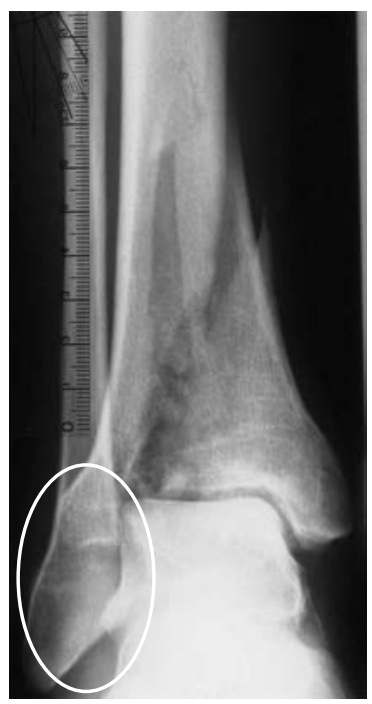

Fig. 3a

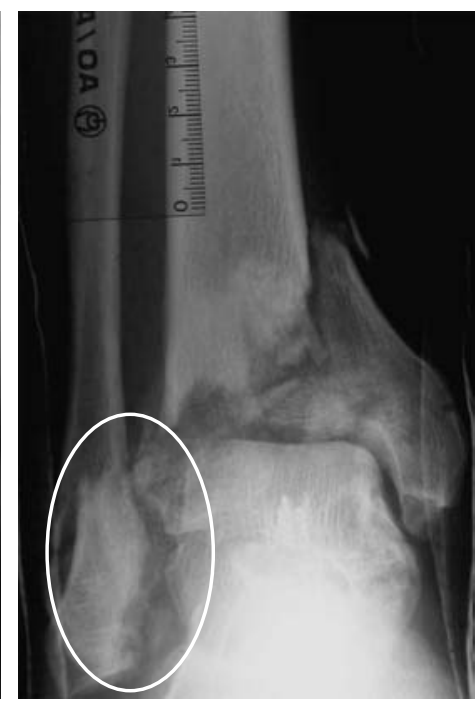

Fig. 3b
Radiographs showing lateral-disruption-type fractures with disruption of the normal talofibular relationship (circled). Figure $3 a-$ Intact lateral column. Figure $3 b-$ Disrupted lateral column.

(anterolateral and posterolateral) resulting in functional diastasis (Fig. 2b) with widening of the mortise and instability of the ankle.

Sixteen cases $(13 \%)$ showed a lateral-disruption-type fracture (Fig. 3), in which the normal relationship between the lateral border of the talus and the distal fibula was disturbed. This finding was strongly associated with an intact fibula; eight of 16 lateral-disruption fractures had an intact 


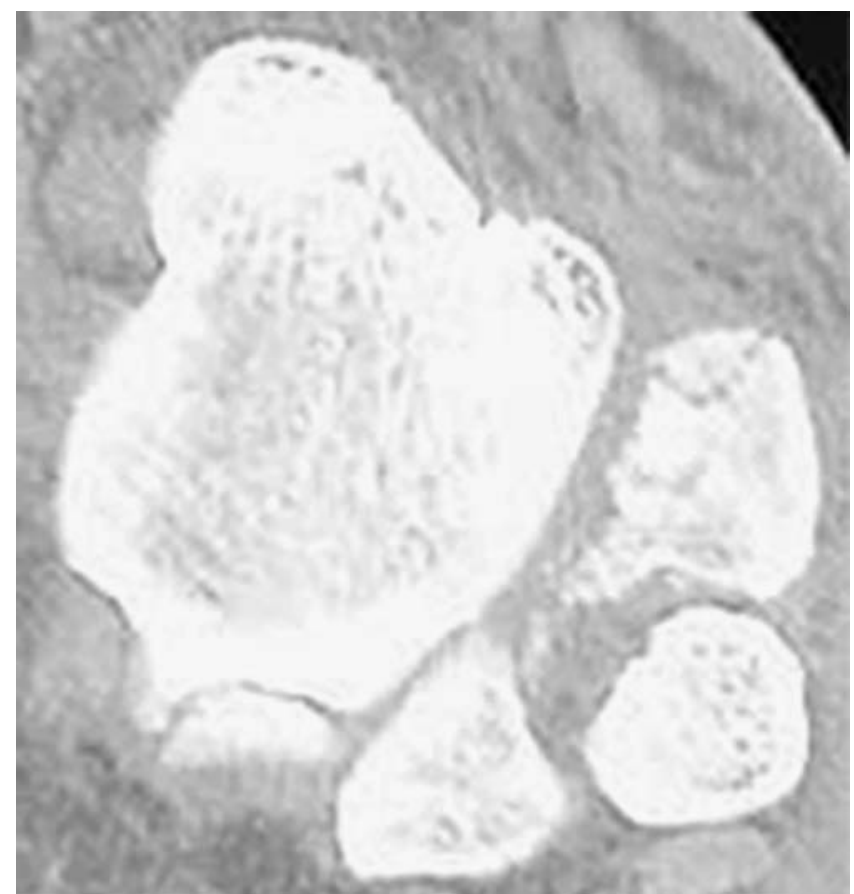

Fig. 4

CT scan of a lateral-disruption-type fracture with maintenance of the normal tibiofibular relationship, the lateral tibial fragments lying lateral to the talus.

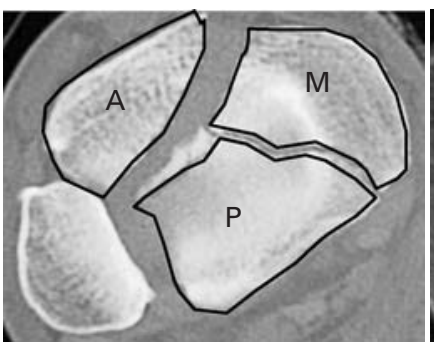

Fig. $5 a$

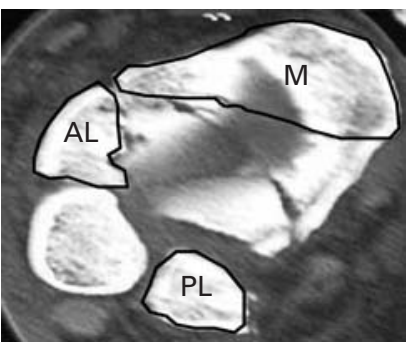

Fig. $5 b$
CT scans of articular fragments showing a) anterior $(A)$, posterior $(P)$, and medial $(M)$ and b) anterolateral $(A L)$ and posterolateral $(P L)$ fragments.

fibula compared with 12 of 108 with a normal talofibular relationship (chi-squared test, $\mathrm{p}<0.0001$ ). A pilon fracture with intact fibulae was therefore eight times more likely to have a disruption of the talofibular joint in this series.

In six of the 16 lateral-disruption fractures, there was an intact lateral column of the tibia (AO, type-B fracture, Fig. $3 a)$. In ten, there was comminution of the lateral tibial column with separation of the tibial shaft from the lateral plafond (AO, type-C fracture, Fig. 3b). The association between an intact fibula and an intact lateral column of the tibia in the lateral-disruption fractures was significant (Fisher's exact test, $\mathrm{p}=0.007$ ).

The relationship of the tibia and fibula in the lateraldisruption fractures was examined by CT in 12 fractures. In

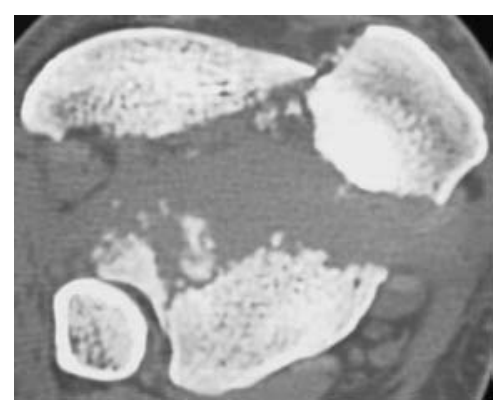

Fig. 6a

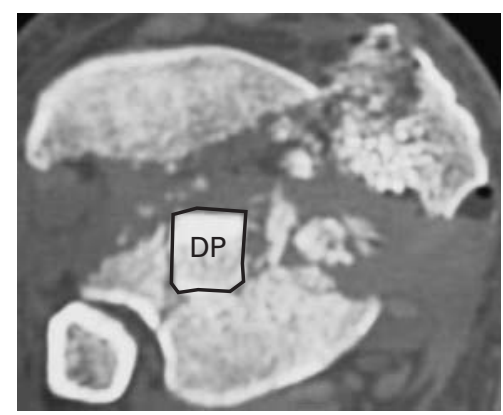

Fig. 6b

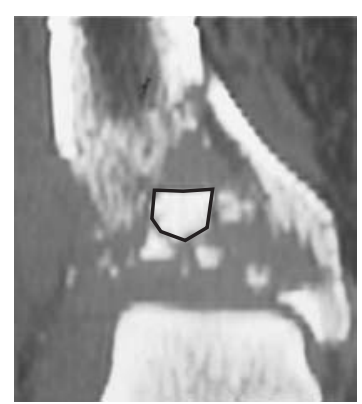

Fig. 6c

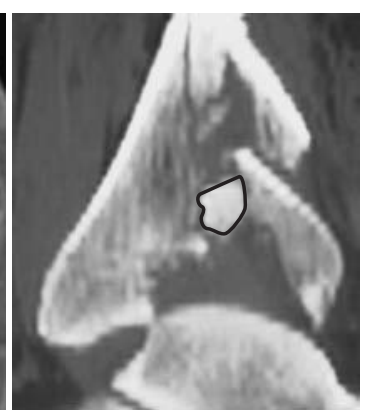

Fig. 6d
CT scans of a Die-punch fragment (DP) showing a) assessment with articular cut, b) position of impaction and c) coronal and d) sagittal reconstructions.

all, a portion of the tibia retained its normal relationship with the distal fibula, namely lateral and inferior to the talar dome (Fig. 4). The fragments retaining the normal relationship were both anterior and posterior in six. In five, the anterior fragment alone maintained its normal relationship with the fibula, a consequence of rupture of the posterior inferior tibiofibular ligament. In one case the posterior fragment alone maintained its normal relationship because of rupture of the anterior inferior tibiofibular ligament.

CT. Fracture fragments. Articular CT scans were available in 108 of the 126 fractures. Six distinct fragments could be recognised although not all were universally present. The major fragments were anterior, posterior, medial, anterolateral, posterolateral (Fig. 5) and die-punch (Fig. 6).

The anterior fragment comprised the bulk of the anterior tibial articular surface and was absent in $14(13 \%)$ fractures in which it was combined either with the posterior (seven) or medial (seven) fragments. The posterior fragment comprised most of the posterior part of the tibial articular surface and was absent in nine ( $8 \%$ ) when it was combined either with the anterior (seven) or medial (two) fragments. The medial fragment represented the attachment of the medial malleolus and contained a variable amount of the horizontal articular surface. It was absent in $22(20 \%)$ fractures when it formed either part of the anterior fragment (six), the posterior fragment (nine) or was bisected (seven). The anterolateral fragment was defined as extending less than $50 \%$ of the width of the plafond and, although small, 


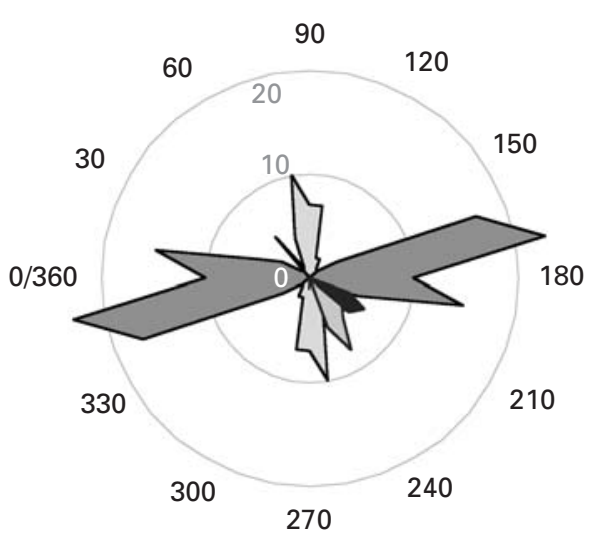

Fig. 7
Radial chart showing the distribution of fracture lines with respect to the intermalleolar axis $\left(0^{\circ}\right.$ to $\left.180^{\circ}\right)$.

Table II. Summary of the types of fracture in each family with numbers

\begin{tabular}{ll}
\hline Coronal family (60) & Sagittal family (36) \\
\hline V type (15) & \\
Y type (23) & T type (20) \\
Anterior split type (8) & Sagittal type (7) \\
Posterior split type (6) & Inverted V type (9) \\
Pure split type (8) & \\
\hline
\end{tabular}

contained the tibial insertion of the anterior inferior tibiofibular ligament. Similarly, the posterolateral fragment extended not more than $50 \%$ across the width of the plafond and represented the tibial insertion of the posterior inferior tibiofibular ligament. The die-punch fragment was central, purely articular and impacted (Fig. 6). Without soft-tissue attachments it could not be reduced by ligamentotaxis.

In some cases there were other fragments. A combined anterior and posterior fragment was named a lateral fragment (see Fig. 8a). A large central articular fragment without impaction was defined as central comminution.
Although this fragment was unlikely to prevent reduction like the die-punch fragment, it was often large enough to require stabilisation. In $22(20 \%)$ fractures, as well as the large distinct fragments, there was evidence of general comminution, distributed among the main articular fragments. The incidence, area and proportion of all fragments were measured.

Fracture families. When the position of the fracture lines was plotted radially (Fig. 7) two major peaks were seen in the sagittal and coronal planes. Analysis with respect to these lines demonstrated two fracture families, the sagittal ( 36 cases; $33 \%$ ) and the coronal (60 cases; 56\%) (Table II). Of the remaining 12 fractures $(11 \%)$ with CT scans, seven $(6 \%)$ were so comminuted that a fracture type could not be attributed to them and five (5\%) had a marginal fracture. Sagittal family (Fig. 8). In seven (19\%) cases there was a simple sagittal split-type fracture (Fig. 8a) with medial and lateral fragments. In nine $(25 \%)$ there was an additional anterolateral fragment creating the inverted V-type fracture (Fig. 8b) and in $20(56 \%)$ the lateral fragment was split coronally, creating a T-type fracture (Fig. 8c). Progression from a sagittal-split-type to a T-type fracture was associated with increasing comminution of the proximal dissociation (Fisher's exact test, $\mathrm{p}=0.023$ ), suggesting an evolution of the sagittal family fractures with increased energy transfer at injury.

Coronal family (Fig. 9). In 22 (37\%) cases there was a single fracture line in the coronal plane. In eight $(13 \%)$, the fracture line followed the intermalleolar axis, bisecting the medial malleolus and thus defining the coronal split-type fracture (Fig. 9a). In eight (13\%), the fracture passed anterior to the medial malleolus creating an anterior split-type fracture (Fig. 9b) and in six (10\%) it was posterior to the medial malleolus, creating the posterior split-type fracture (Fig. 9c). Thirty-eight (63\%) cases had two broadly coronal fracture lines passing anterior and posterior to the intermalleolar axis thereby separating the medial fragment as a triangular portion of the horizontal articular surface. In 15 $(25 \%)$ cases the fracture lines diverged at the lateral edge of the plafond creating the V-type fracture (Fig. 9d) and in 23 $(38 \%)$, the separation occurred centrally, describing the Ytype fracture (Fig. 9e).

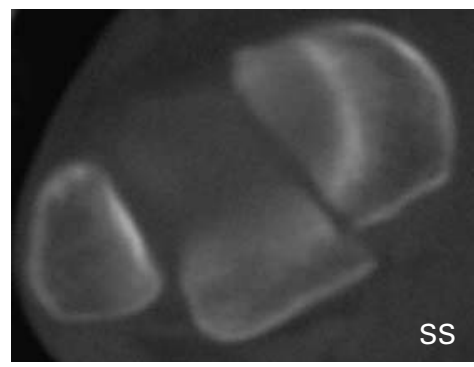

Fig. 8a

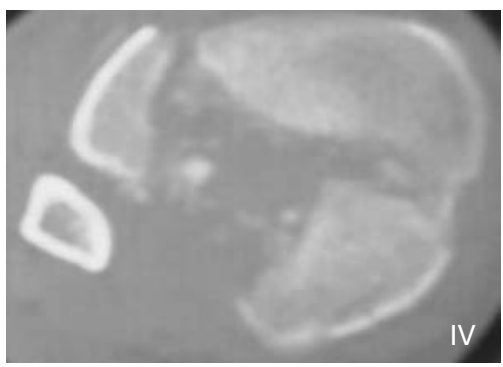

Fig. 8b

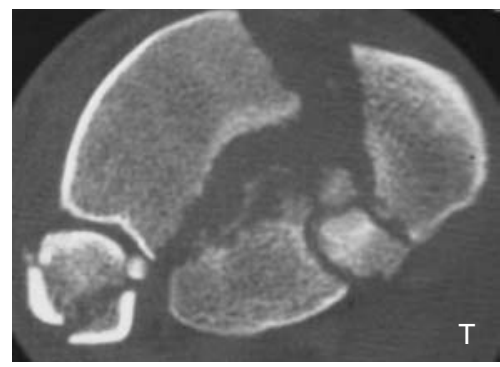

Fig. 8c

CT scans of sagittal family fractures showing a) the sagittal split (SS), b) inverted V (IV) and c) T types of fractures. 


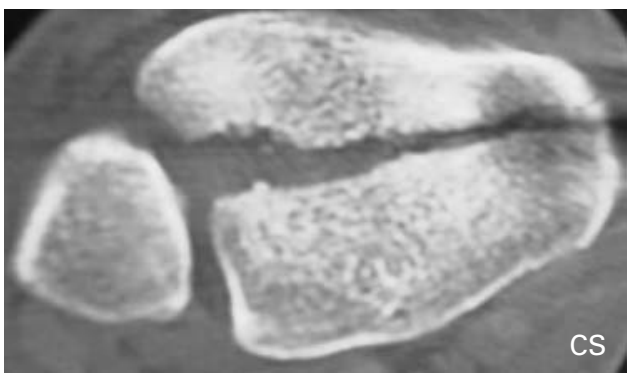

Fig. 9a

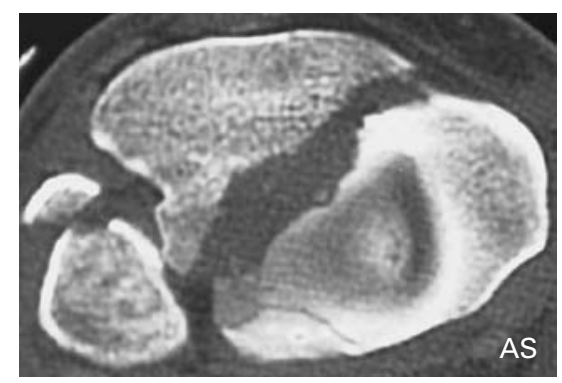

Fig. 9b

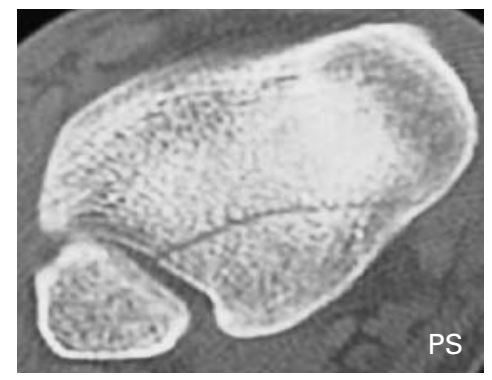

Fig. 9c

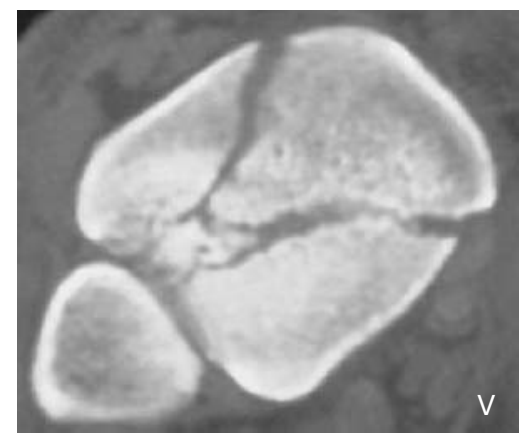

Fig. 9d

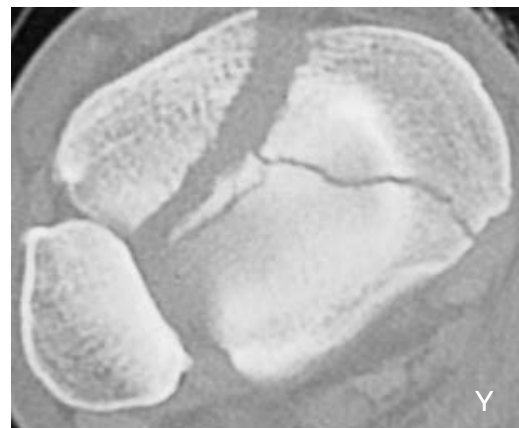

Fig. 9e
CT scans of coronal family fractures showing a) the coronal split (CS), b) the anterior split (AS), c) the posterior split (PS), d) the $\mathrm{V}$ and e) the $\mathrm{Y}$ types of fracture.
The coronal fracture line was significantly more anterior in the $\mathrm{Y}$ and anterior split types compared with the $\mathrm{V}$ and posterior split types (Kruskal Wallis test, $\mathrm{p}=0.0024$ ). The height of fall to produce the fractures also showed significant differences between the types (ANOVA, $\mathrm{p}=0.01$ ); $\mathrm{Y}$ and anterior split fractures resulting from higher falls than $\mathrm{V}$ and posterior split types respectively, with a progression within the two groups from simple to comminuted. These findings suggest an evolution of the coronal family fractures with increasing energy transfer, the posterior split fractures becoming $\mathrm{V}$ type and the anterior split becoming $\mathrm{Y}$ type with progressive energy transfer at injury.

Sagittal fractures tended to present in varus, had a more proximal metaphyseal/diaphyseal dissociation and followed a higher energy injury in younger patients. Coronal fractures presented in valgus with a more distal dissociation, following lower energy trauma in older patients.

There was no association between the lateral-disruption fracture and fracture family, but lateral-disruption fractures were comminuted (Fisher's exact test, $\mathrm{p}=0.006$ ). The comminuted fractures were 14.7 times more likely to have a lateral disruption compared with sagittal and coronal family fractures.

Reproducibiity of fracture descriptions. Using the AO classification, the average kappa value among the five reviewers for AO type-B or type-C fractures was 0.4637 ; for the Rüedi and Allgöwer ${ }^{7}$ system it was 0.4522 , suggesting that these traditional systems are poorly reproducible. Interobserver agreement using our new articular fracture description gave a mean kappa value of 0.666 for fracture family and 0.5764 across the ten types of fracture. Intra- observer agreement performed by the first author gave a mean kappa value of 0.8616 . This suggests that the descriptions of fractures in our study are superior in their reproducibility.

\section{Discussion}

The pilon fracture has a number of components, namely, the proximal extension, the fracture of the fibula with its relationships and, probably the most important, the articular fracture. Although all parts are associated, the problems which they present are different and it is logical therefore to consider them separately.

Our analysis has implications for the choice of implant. Simple fractures can be stabilised by interfragmentary lag screws, whereas more comminuted types and those extending into the diaphysis require more robust methods of stabilisation because these patterns have increased rates of malunion and nonunion. ${ }^{1}$

The most proximal extent of the fracture in this series was $16 \mathrm{~cm}$ from the plafond. If a plate is used it must be long enough to achieve adequate proximal purchase. After fixation of the articular fracture, usually achieved with 3.5 $\mathrm{mm}$ screws, there must remain sufficient space within the articular segment to allow proximal stabilisation. Three crossed fine wires can be placed above or below the articular fragment screws and take a maximum of $6 \mathrm{~mm}$ to provide stabilisation, but fixation with a plate may require significantly more space proximal to the screws in the articular fragment. Finally, it cannot be assumed that all fractures will be optimally stabilised by a medial plate. In $60(55 \%)$ of our cases with coronal fracture lines, this 


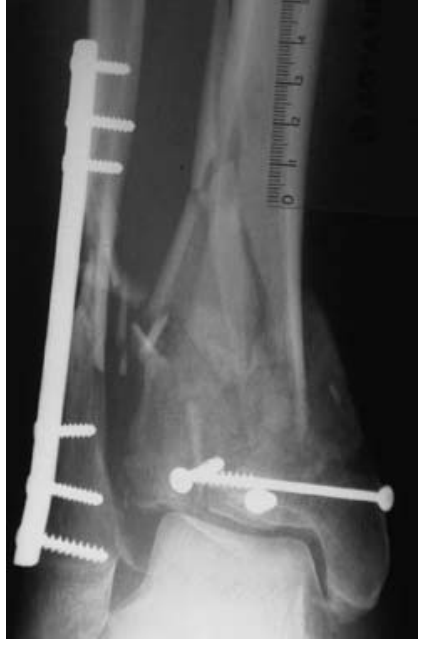

Fig. 10a

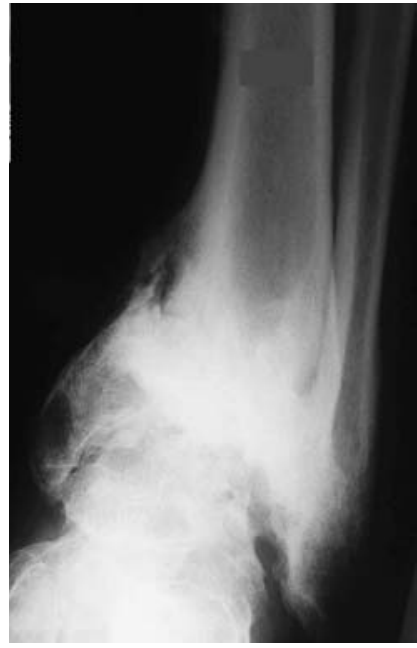

Fig. $10 \mathrm{~b}$
Radiographs of examples of failure to recognise lateral-disruption fractures a) after initial fixation and b) showing the long-term result after the onset of osteoarthritis.

would potentially result in screws interfering with the fracture.

The relationship between the tibia and the fibula is normally described by the state of the syndesmosis. We identified one ligamentous diastasis and 52 fractures (44\%) in which there was disruption of the normal tibiofibular relationship through bone. Failure to recognise this 'functional diastasis' with inadequate reduction of the lateral tibial fragments could result in a widened mortise and instability.

Similarly without restoration of the normal talofibular relationship in lateral-disruption fractures, the function of the ankle will inevitably be compromised (Fig. 10). This restoration will automatically reduce the lateral tibial fragments, thus providing a key for fixation of the remaining articular surface.

Within each fracture group there was progression from a simple to a more complex type with increasing transfer of energy. In most fractures the fibular fracture was an early occurrence, whereas in the lateral-disruption group it was a late event. Whether this evolution is related to the energy of injury or is purely directional cannot be determined from our data.

Our study was dependent on CT which is essential in the management of pilon fractures of the distal tibia. Since the publication of the Rüedi and Allgöwer classification ${ }^{7}$ it has been accepted that the defining feature of the fracture of the articular surface is a coronal fracture line. In our series this is not the case in $33 \%$ of cases with a sagittal family fracture. This has implications for treatment because, although the fracture can be approached directly from the anterior aspect, fixation screws will need to be placed percutaneously in the coronal direction.

We have introduced two distinct fracture families which appear to be influenced by the degree of transfer of energy at the time of injury, the direction of the force and to a less degree, the age of the patient. There also appears to be a progression of the injury of fracture within each family, again dependent on transfer of energy. The sagittal family fractures occur after higher energy with varus angulation in younger patients while the coronal family fractures occur with valgus angulation in older patients after less severe trauma. The coronal alignment may relate to the attitude of the foot at impact or it may be due to weakness of tibialis posterior with increasing age.

If a description of the fracture is to be valuable, it must be reproducible. Levels of agreement within the AO and Rüedi and Allgöwer classification systems are at best adequate, a finding supported by other groups. ${ }^{8-10}$ By contrast, the level of agreement with our description of the pilon fracture is good to excellent.

We would like to acknowledge the pilot work done for this study performed by Mr Joe Sudhaker and Mr Duncan Whitwell; Mrs Rosemary Greenwood for her guidance in the statistical methods and the Secretarial, Clinic and Radiology Staff at the Bristol Royal Infirmary.

The author or one or more of the authors have received or will receive benefits for personal or professional use from a commercial party related directly or indirectly to the subject of this article. In addition, benefits may have been or will be directed to a research fund, foundation, educational institution, or other non-profit organisation with which one or more of the authors are associated.

\section{References}

1. Heim U. The pilon tibial fracture: classification, surgical techniques, results. Philadelphia: W. B. Saunders Co., 1995.

2. Müller ME, Allgöwer M, Schneider R, Willenegger H. Manual of internal fixation. Second ed. Berlin, etc: Springer-Verlag, 1979.

3. Ashurst APC, Bromer RS. Classification and mechanism of fractures of the leg bones involving the ankle. Arch Surg 1922;4:51-129.

4. Bonnin JG. Injuries to the ankle. London: Heinermann, 1950.

5. Cyr L, Francis K. Measures of clinical agreement for nominal and categorical data: the kappa coefficient. Comp Biol Med 1992;22:239-46.

6. Altman DG. Practical statistics for medical research. First ed. London: Chapman \& Hall, 1991.

7. Rüedi TP, Allgöwer M. The operative treatment of intra-articular fractures of the lower end of the tibia. Clin Orthop 1979;138:105-10.

8. Martin JS, Marsh JL, Bonar SK, et al. Assessment of the AO/ASIF fracture classification for the distal tibia. J Orthop Trauma 1997;11:477-83.

9. Swiontkowski MF, Sands AK, Agel J, et al. Interobserver variation in the AO/OTA fracture classification system for pilon fractures: is there a problem? J Orthop Trauma 1997; 11:467-70.

10. DirschI DR, Adams GL. A critical assessment of factors influencing reliability in the classification of fractures, using fractures of the tibial plafond as a model. J Orthop Trauma 1997;11:471-6

11. Gustilo RB, Anderson JT. Prevention of infection in the treatment of one thousand and twenty five open fractures of long bones: retrospective and prospective analyses. J Bone Joint Surg [Am] 1976;58-A:453-8.

12. Gustilo RB, Mendoza RM, Williams DN. Problems in the management of type III (severe) open fractures: a new classification of type III open fractures. J Trauma 1984;24:742-6.

13. Müller ME, Nazarian S, Koch P, Schatzker J. The comprehensive classification of fractures of long bones. Berlin: Springer-Verlag, 1990. 